\title{
An Arab Adolescent with Multisystem Inflammatory Syndrome Associated with COVID-19: A Report from Syria
}

\author{
Jaafer Zaino ${ }^{1}$ Abdullah Bakri ${ }^{1} \quad$ Amani Al Sayed Ahmad ${ }^{1} \quad$ Ghadeer Hadba ${ }^{1}$ \\ ${ }^{1}$ Intensive Care Unit, Syrian Mouassat Association Hospital, \\ Damascus, Syria \\ Address for correspondence Ghadeer Hadba, MD, Intensive Care \\ Unit, Syrian Mouassat Association Hospital, Damascus, Syria \\ (e-mail: ghadeer_hadba@hotmail.com). \\ Avicenna J Med 2021;11:221-224.
}

\author{
Abstract \\ Keywords \\ - arab \\ - coronavirus disease \\ 2019 (COVID-19) \\ - kawasaki disease \\ - multisystem \\ inflammatory \\ syndrome in children \\ (MIS-C) \\ - severe acute \\ respiratory syndrome \\ coronavirus 2 (SARS- \\ CoV-2)
}

Multisystem inflammatory syndrome in children (MIS-C) is a rare and critical condition that affects children following exposure to severe acute respiratory syndrome Coronavirus 2 (SARS-CoV-2) infection, leading to multiorgan dysfunction and shock. MIS-C has been reported from different parts of the world but rarely from Arab countries. In this report, we describe a 15-year-old Arab boy who was admitted to the ICU during the surge of Coronavirus transmission in Syria with a clinical picture consistent with MIS-C, including high-grade fever, gastrointestinal symptoms, rash, multiorgan dysfunction, and shock. Laboratory profile showed significant elevation of inflammatory markers, negative SARS-CoV-2 RT-PCR testing but positive serologic testing for SARS-CoV-2. The patient received intravenous immunoglobulins (IVIG) and glucocorticoids with remarkable cardiac improvement and significant alleviation in inflammatory markers. To our knowledge, this is the first reported case of MIS-C from Syria, which adds to the epidemiological data about this new syndrome.

\section{Introduction}

Although acute respiratory syndrome Coronavirus 2 (SARSCoV-2) infection in children is usually mild or even asymptomatic, several reports of multisystem inflammatory syndrome in children (MIS-C) have called physicians to be alert for this severe and life-threatening condition. ${ }^{1,2}$ MIS-C is a rare and critical condition occurring predominantly in previously healthy children following exposure to SARS-CoV-2 infection, leading to multiorgan dysfunction and shock. MIS$\mathrm{C}$ has been reported from different parts of the world but rarely from Arab countries. ${ }^{3}$ In this report, we describe the first reported case of MIS-C from Syria in a 15-year-old Arab boy who initially complained of fever, abdominal pain and diarrhea, but later he developed multiorgan dysfunction and fluid-unresponsive shock requiring vasopressors and ICU admission.

\section{Case History}

A 15-year-old male without previous illnesses, who is fully vaccinated (including bacillus Calmette-Guerin [BCG]), presented to the emergency room complaining of persistent high-grade fever, generalized abdominal pain, nausea, vomiting, and watery diarrhea, which started in the past 6 days along with malaise and headache. Minimal cough, dyspnea, and sore throat were mentioned. Noticeably, local surge of SARS-CoV-2 transmission was recorded at the time of his published online

September 16, 2021
DOI https://doi.org/ $10.1055 / \mathrm{s}-0041-1734387$. ISSN 2231-0770.

\footnotetext{
(c) 2021. Syrian American Medical Society. All rights reserved. This is an open access article published by Thieme under the terms of the Creative Commons Attribution-NonDerivative-NonCommercial-License, permitting copying and reproduction so long as the original work is given appropriate credit. Contents may not be used for commercial purposes, or adapted, remixed, transformed or built upon. (https://creativecommons.org/ licenses/by-nc-nd/4.0/) Thieme Medical and Scientific Publishers Pvt. Ltd., A-12, 2nd Floor, Sector 2, Noida-201301 UP, India
} 


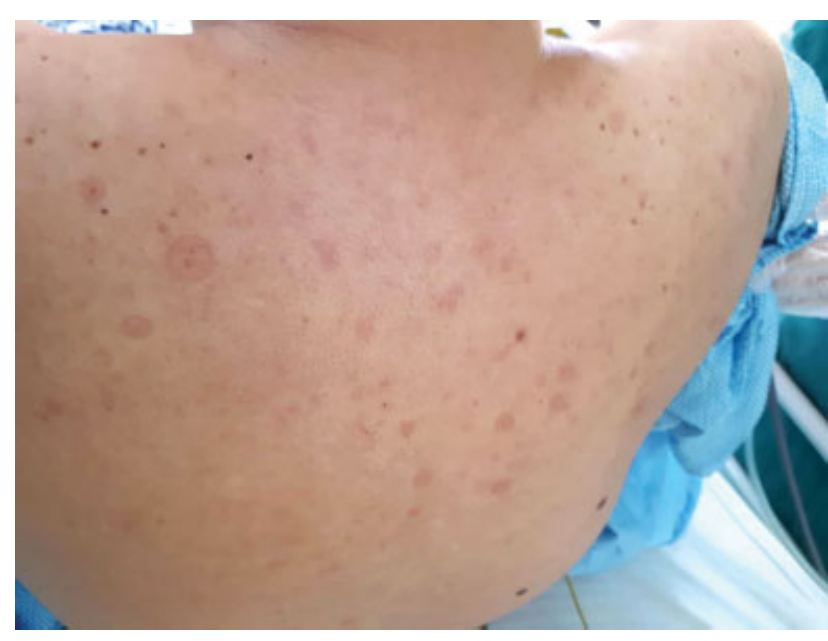

Fig. 1 Erythematous macular rash.

presentation, and his parents along with his younger sister reported they had mild Coronavirus disease 2019 (COVID19) 2 weeks earlier.

On physical examination, he was fully alert but modestly ill, with blood pressure $(70 / 40 \mathrm{~mm} \mathrm{Hg}$ ), heart rate (120/min), temperature $\left(39^{\circ} \mathrm{C}\right)$, respiratory rate $(26 \mathrm{~b} / \mathrm{min})$, oxygen saturation (92\% in room air), height $(170 \mathrm{~cm})$, and weight $(75 \mathrm{~kg})$. He was admitted to ICU because of hypotension. We noticed bilateral nonexudative conjunctivitis, strawberry tongue, and erythematous macular rash on upper body (-Fig. 1). Lung auscultation was clear at presentation, but later he developed bilateral basal fine crackles. Tender hepatomegaly and palpable spleen were evident. No neck stiffness, neck lymphadenopathy, ankle edema, jaundice, or cardiac murmurs were present.

Chest X-ray showed enlarged cardiopulmonary index without pulmonary infiltrates. Electrocardiogram revealed sinus tachycardia with poor $\mathrm{R}$ wave progression without signs of acute ischemia. Chest CT without contrast demonstrated mediastinal lymphadenopathy, small pleural effusion, and dorsal lower lobes micronodules bilaterally. Abdominal ultrasound demonstrated moderate hepatosplenomegaly, mesenteric adenitis, and slight ascitic fluid. Transthoracic echocardiogram confirmed dilated cardiomyopathy, impaired ejection fraction ( $E F=40 \%$ ), mild pulmonary hypertension, and small pericardial effusion without valvular disorders.

Notable laboratory findings included the following: white blood cells $17570(4500-10500 / \mu \mathrm{L})$ with left shift and lymphopenia $500 / \mu \mathrm{L}$, platelets $55(150-450 \mathrm{~K} / \mu \mathrm{L})$, hemoglobin $11.8(12-17.4 \mathrm{~g} / \mathrm{L})$, urea $38(13-43 \mathrm{mg} / \mathrm{dl})$, creatinine 1.4 (0.6-1.3 mg/dl), albumin 2.02 (3.5-5.5 g/dl), aspartate transaminase 56 (1-40 U/L), alanine transaminase 32 (1-42 U/L), sodium 130 (135-145 mmol/L), potassium 2.95 (3.5-5.1 $\mathrm{mmol} / \mathrm{L}), \quad$-reactive protein (CRP) $15.25(0.0-0.5 \mathrm{mg} / \mathrm{dl})$, procalcitonin $>100(0.5-2.0 \mathrm{ng} / \mathrm{ml})$, ferritin 468 (30$400 \mathrm{ng} / \mathrm{ml}$ ), prothrombin time 24.3 (12.5 Sec), PTT 52.9 (24-39 Sec), D-dimer $9.25(0.0-0.5 \mathrm{mg} / \mathrm{L})$, fibrinogen 396 (160-400 mg/dl), troponin-I $0.85(0-0.06 \mathrm{ng} / \mathrm{ml})$, lactate dehydrogenase 577 (230-460 U/1), creatine kinase 198 (24-195
$\mathrm{U} / \mathrm{l})$, triglyceride $181(50-150 \mathrm{mg} / \mathrm{dl}$ ), blood group (B+), negative cytomegalovirus and Epstein-Barr virus IgM antibodies, negative SARS-CoV-2 reverse transcriptase polymerase chain reaction (RT-PCR) testing of nasopharyngeal swab, negative SARS-CoV-2 IgM antibodies but positive SARSCoV-2 IgG antibodies, and 17.89 cutoff index (COI; 1.0). No other respiratory viruses were tested.

Noradrenaline was initiated after that $2 \mathrm{~L}$ of normal saline failed to normalize blood pressure. Wide-spectrum antibiotics (meropenem and vancomycin) were given but stopped later after negative blood culture. Due to high suspicion of MIS-C, he received dexamethasone ( $8 \mathrm{mg}$ three times daily until discharge), intravenous immunoglobulins (IVIG) $2 \mathrm{~g} / \mathrm{kg}$ as a single dose infused over 12 hours, low-dose aspirin ( $75 \mathrm{mg}$ once daily, initiated on the third day of admission after his platelets counted $>100 \mathrm{~K} / \mu \mathrm{l}$ ), enoxaparin ( $40 \mathrm{mg}$ twice daily), in addition to furosemide and oxygen therapy. After which, the patient was rendered afebrile and hemodynamically stable. The patient continued to improve and was discharged after 8 days of hospitalization, including 5 days of ICU stay. On discharge, follow-up transthoracic echocardiography showed improved left ventricular function ( $E F=50 \%$ ). Biochemically, all of C-reactive protein, platelets, renal function, and coagulopathy recovered. Hepatosplenomegaly decreased slightly on discharge. Follow-up transthoracic echocardiogram and laboratory assessment were performed 2 weeks later, which revealed normal D-dimer, troponin and inflammatory biomarkers in addition to normal left ventricular function ( $E F=60 \%$ ) without evidence of coronary aneurysm, and aspirin was stopped. Hepatosplenomegaly disappeared.

\section{Discussion}

The clinical picture of this patient meets The American Academy of Pediatrics (AAP) criteria for MIS-C. ${ }^{4}$ It is consistent with previous reports of MIS-C from different parts of the world, which occurred predominantly in previously healthy children and adolescents. ${ }^{5}$ In agreement with these reports, gastrointestinal symptoms and persistent highgrade fever were the presenting complaints of our patient with considerably few respiratory symptoms. Hepatosplenomegaly and mesenteric adenitis were remarkably evident. Cardiac dysfunction along with vasopressor requiring shock, acute kidney injury, coagulopathy, and significantly elevated inflammatory markers made hyperinflammatory multiorgan dysfunction the likely explanation. Besides MIS-C, the main differential diagnosis included toxic shock syndrome, macrophage activation syndrome, and Kawasaki disease (KD). However, the accompanying wave of Coronavirus community transmission in Syria at the time of our patient's presentation in mid-August and his family history of COVID19 supported MIS-C highly. ${ }^{6}$ What makes a child with COVID-19 at risk of or susceptible to MIS-C is still unknown. In addition, the pathophysiology behind this syndrome is currently unclear, although a postinfectious immunological process in predisposed children rather than a direct viral infection is already postulated. ${ }^{7}$ This is supported by the fact 
that the majority of recorded cases have negative SARS-CoV2 RT-PCR testing but positive serologic testing for SARS-CoV$2 .^{8}$ Despite the similarity between KD and MIS-C, epidemiological data imply that they are separate entities. ${ }^{8}$ However, a link between the two diseases cannot be currently refuted. Immunomodulatory agents are the mainstay of management for MIS-C. Our patient received IVIG and glucocorticoids with remarkable cardiac improvement and significant alleviation in inflammatory markers. Whether MIS-C has long-term complications is not already established, which means that close follow-up is warranted. To our knowledge, this is, to date, the first reported case of a Syrian Arab adolescent with MIS-C, which adds to the epidemiological information about this new syndrome.

\section{Conflict of Interest}

None declared.

\section{References}

1 Dong Y, Mo X, Hu Y, et al. Epidemiology of COVID-19 among children in China. Pediatrics 2020;145(06):e20200702

2 Centers for Disease Control and Prevention. Multisystem inflammatory syndrome in children (MIS-C) associated with Coronavi- rus disease. Available at: https://emergency.cdc.gov/han/2020/ han00432.asp. Accessed September 23, 2020.

3 Aronoff SC, Hall A, Del Vecchio MT. The natural history of SARSCov-2 related multisystem inflammatory syndrome in children (MIS-C): a systematic review. J Pediatr Infect Dis Soc 2020;9(06): 746-751

4 American Academy of Pediatrics clinical guidance: multisystem inflammatory syndrome in children (MIS-C). Available at: https:// services.aap.org/en/pages/2019-novel-coronavirus-covid19-infections/clinical-guidance/multisystem-inflammatory-syndrome-in-children-mis-c-interim-guidance/. Accessed June 9, 2021

5 Feldstein LR, Rose EB, Horwitz SM, et al.Overcoming COVID-19 Investigators; CDC COVID-19 Response Team. Multisystem inflammatory syndrome in U.S. children and adolescents. N Engl J Med 2020;383(04):334-346

6 World Health Organization. WHO Coronavirus Disease (COVID-19) Dashboard. Available at: https://covid19.who.int/region/emro/ country/sy. Accessed September 23, 2020

7 Jiang L, Tang K, Levin M, et al. COVID-19 and multisystem inflammatory syndrome in children and adolescents. Lancet Infect Dis 2020;20(11):e276-e288

8 Whittaker E, Bamford A, Kenny J, et al. PIMS-TS Study Group and EUCLIDS and PERFORM Consortia. Clinical characteristics of 58 children with a pediatric inflammatory multisystem syndrome temporally associated with SARS-CoV-2. JAMA 2020;324(03): 259-269 